\title{
Radiation Protection
}

Correspondence to Young-Yong $\mathrm{Ji}$ yyji@kaeri.re.kr

\section{Development and Performance of a Hand-Held CZT Detector for In-Situ Measurements at the Emergency Response}

\author{
Young-Yong Ji*, Kun Ho Chung*, Chang-Jong Kim*, Jin Yoon ${ }^{\dagger}$, Wanno Lee*, Geun-Sik Choi*, \\ and Mun Ja Kang* \\ *Korea Atomic Energy Research Institute, Daejeon, Republic of Korea \\ ${ }^{+}$SI Detection Co. Ltd, Daejeon, Republic of Korea
}

\begin{abstract}
Background: A hand-held detector for an emergency response was developed for nuclide identification and to estimate the information of the ambient dose rate in the scene of an accident as well as the radioactivity of the contaminants.

Materials and Methods: To achieve this, the most suitable sensor was first selected as a cadmium zinc telluride (CZT) semiconductor and the signal processing unit from a sensor and the signal discrimination and storage unit were successfully manufactured on a printed circuit board.

Results and Discussion: The performance of the developed signal processing unit was then evaluated to have an energy resolution of about $14 \mathrm{keV}$ at $662 \mathrm{keV}$. The system control unit was also designed to operate the CZT detector, monitor the detector, battery, and interface status, and check and transmit the measured results of the ambient dose rate and radioactivity. In addition, a collimator, which can control the inner radius, and the airborne dust sampler, which consists of an air filter and charcoal filter, were developed and mounted to the developed CZT detector for the quick and efficient response of a nuclear accident.

Conclusion: The hand-held CZT detector was developed to make the in-situ gamma-ray spectrometry and its performance was checked to have a good energy resolution. In addition, the collimator and the airborne dust sampler were developed and mounted to the developed CZT detector for a quick and efficient response to a nuclear accident.
\end{abstract}

Keywords: In-situ measurement, Hand-held detector, CZT, Signal processing, Radioactivity

This is an Open-Access article distributed under the terms of the Creative Commons Attribution Non- Commercial License (http://creativecommons.org/licenses/by-nc/3.0) which permits unrestricted non-commercial use, distribution, and reproduction in any medium, provided the original work is properly cited. 


\section{INTRODUCTION}

The emergency responses during a nuclear accident demands information regarding the ambient dose rate and deposited radionuclides on the ground. In particular, rapid determinations with the in-situ measurement are indispensable in the early phase to estimate the amount released and design a response strategy. Since a portable high purity Ge (HPGe) detector is usually used to survey deposited gamma nuclides, the aspect of its cooling and collimation can hinder a rapid response. The advent of a hand-held detector in which the energy resolution is sufficient to identify the detected gamma nuclides and cooling is not necessary provide great efficiency for an in-situ measurement during an emergency response. A cadmium zinc telluride (CZT) detector, which is a compound semiconductor consisting of cadmium, zinc and telluride, has a good resolution, requires no cooling, and has a high dose rate capability [1-3]. Therefore, a CZT detector can be selected to be the most suitable detector for the spectroscopic identification of gamma nuclides during an emergency response even with a very high count rate at room temperature.

In this study, a hand-held CZT detector was developed using a CZT sensor with a volume of $0.5 \mathrm{~cm}^{3}$ (CZT500S, GBS Elektronik GmbH, Groberkmannsdorf, Germany), and making up the signal processing circuits with an on-board type. First, a detector body with a hand-held type was designed to be divided into two parts, the signal process and the system control. A preamplifier for the pulse shaping from a CZT sensor was modified so as to be connected to the printed circuit board (PCB) with several circuits for the signal processing, such as an amplifier, pile-up rejection, pole-zero cancellation, base line restoration, and multichannel analyzer. These components were inserted into a cylindrical detector body, and the performance was then evaluated to have a good energy resolution of about $14 \mathrm{keV}$ at $662 \mathrm{keV}$. In the system control part, the GPS (global positioning system), internal battery and DC (direct current) input, LCD (liquid crystal display), control buttons, and several interfaces, such as Bluetooth, USB, and RS232, were arranged with the on-board type. In addition, a collimator [4], which can control the inner radius, and the airborne dust sampler, which consists of an air filter and charcoal filter, were developed and mounted to the developed CZT detector for the quick and efficient response of a nuclear accident (Korean patent 10-1482016; Collimator for radia-

Corresponding author : Young-Yong Ji, yyji@kaeri.re.kr 989-111 Daedeok-daero, Yuseong, Daejeon 305-353, Korea tion detector).

\section{MATERIALS AND METHODS}

\subsection{CZT semiconductor}

An in-situ gamma-ray spectrometer for an emergency response should place an emphasis on the portability with a small size for quick and efficient operation, although it would be an antithetic concept with respect to the radiation detection with a good resolution and efficiency. In general, a CZT is known to have a good resolution with about a $4.64 \mathrm{eV} \mathrm{W}$-value, wide band gap of about $1.57 \mathrm{eV}$ without cooling required, and a small volume. Therefore, the unique combination of spectroscopy and very high count rate capability at room temperature can make a CZT semiconductor as an ideal detector for the emergency response.

\subsection{CZT sensor and preamplifier}

A CZT with a volume of $1 \times 1 \times 0.5 \mathrm{~cm}^{3}$ was selected to be an optimized sensor to achieve the information on the dose rate and the radioactivity due to the contaminants, such as ${ }^{131} \mathrm{I},{ }^{134} \mathrm{Cs}$, and ${ }^{137} \mathrm{Cs}$ in a nuclear accident response. The CZT sensor and preamplifier with specifications of an energy resolution of below $18 \mathrm{keV}$ at $662 \mathrm{keV}$ and a signal rise time of below $200 \mathrm{~ns}$ at $50 \mathrm{pF}$, respectively, were used to make the hand-held detector.

\subsection{Modification of a preamplifier}

To compose the signal processing circuits with an on-board type, the output of a preamplifier was modified to connect the PCB, as shown in Fig. 1. The cable connections from its output should be designed to supply high voltage for the CZT sensor operation and low voltage to the preamplifier operation and to output the sensor signal. After modifying the output of the

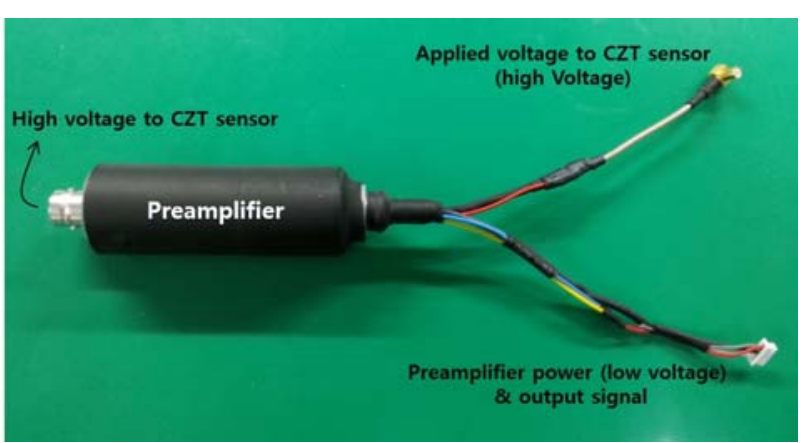

Fig. 1. Modified output of a preamplifier. 


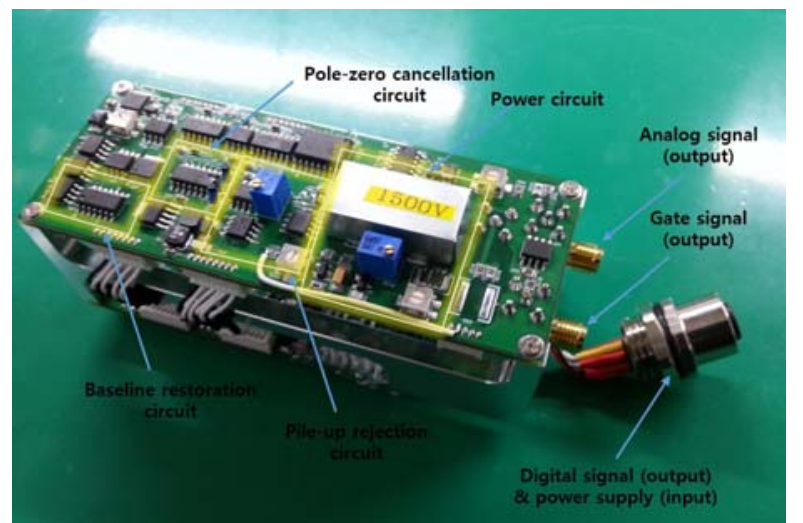

Fig. 2. Developed signal processing unit with an on-board type.

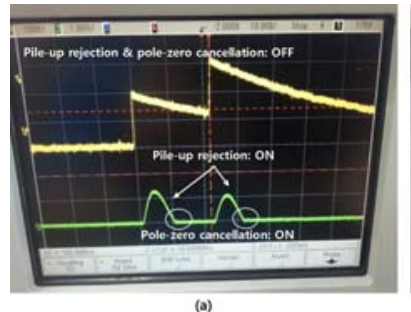

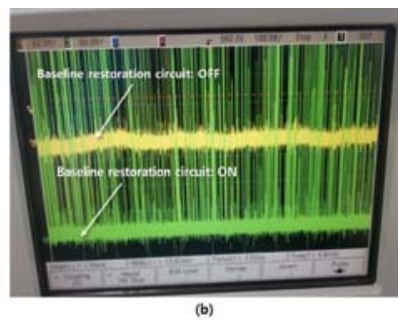

Fig. 3. Signal processing effect due to (a) the pole-zero cancellation and pile-up rejection and (b) the baseline restoration.

preamplifier, its performance was checked from the measurement of the rise time using a point source of ${ }^{137} \mathrm{Cs}$. There was no degradation in the performance for the combination of the CZT sensor and modified preamplifier.

\section{RESULTS AND DISCUSSION}

\subsection{Signal processing unit}

In general, a signal processing unit in a radiation detector means an amplifier and plays the role of pulse shaping and amplifying. It consists of several circuits for the pile-up rejection, pole-zero cancellation, baseline restoration, and amplification, and directly affects the performance parameters as a radiation detector, such as the resolution and efficiency [5]. As shown in Fig. 2, the signal processing board was manufactured by arranging the amplifier circuits, the power circuit, and interfaces for the analog and gate signal.

Since the output of a preamplifier has a long decay time of about 10 to $200 \mu$ s for the sufficient collection of charges produced in a sensor, the pile-up of pulses can be shown by increasing the input count rate in a sensor. In addition, the pulse can undershoot the baseline after the pulse shaping and the baseline can also be fluctuated. They directly impact the resolution of pulses in the measured energy spectrum. Fig. 3 shows the results of the developed signal processing unit from a point source of ${ }^{60} \mathrm{Co}$ just in front of a CZT sensor through the solutions using the pole-zero cancellation, pile-up rejection, and baseline restoration circuit.

\subsection{Signal discrimination and storage}

After the signal processing unit, the output from an amplifier is sent to the multichannel analyzer (MCA), which consists of an analog-to-digital convertor (ADC), CPU, and memory, to discriminate the pulse height and store it according to the its value representing the pulse height. Fig. 4 shows the developed the signal discrimination and storage unit, which is located under the signal processing unit. In its PCB part, the $\mathrm{TxD}$ (transmit data) and $\mathrm{RxD}$ (receive data) circuit was added to transmit and receive the data and the interface was then built on the board to communicate the digital signal and supply the power to the power circuit in the signal processing unit. A modified preamplifier is inserted into the space between two boards for the compact design of the hand-held CZT detector.

These components including the signal processing unit were inserted into a cylindrical detector body, as shown in Fig. 5, and the performance was then evaluated from the measurement of the energy resolution. Fig. 6 shows the energy spectrum for a point source of ${ }^{137} \mathrm{Cs}$ from the developed CZT detector. The full energy absorption peak of gamma-rays from ${ }^{137} \mathrm{Cs}$ was measured with a good energy resolution of about 14 $\mathrm{keV}$ at $662 \mathrm{keV}$ and two $\mathrm{K}$ x-ray peaks of about 32 and $36 \mathrm{keV}$ from ${ }^{137} \mathrm{Ba}$ were also measured in the energy spectrum of ${ }^{137} \mathrm{Cs}$. The results of the measurement of the energy resolution using several point sources are shown in Fig. 7. Since the energy resolution was well defined until the photon energy of about 1.0 $\mathrm{MeV}$, the developed CZT detector was sufficient to

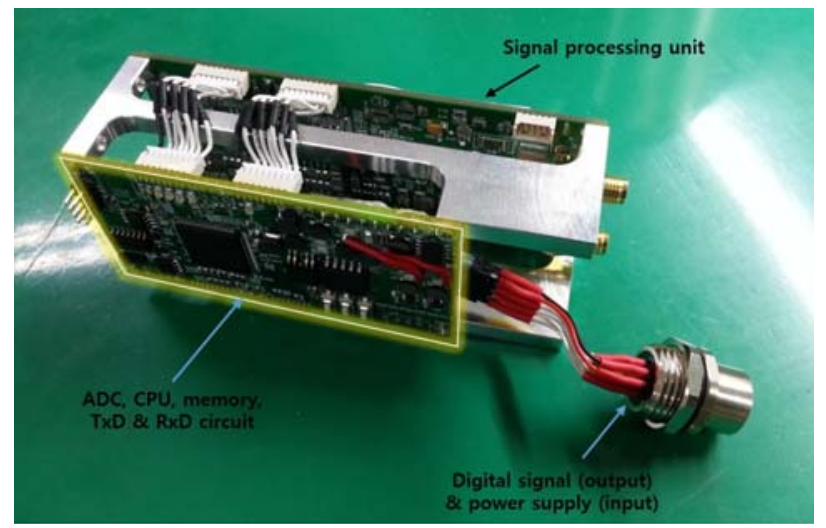

Fig. 4. Developed signal discrimination and storage unit. 


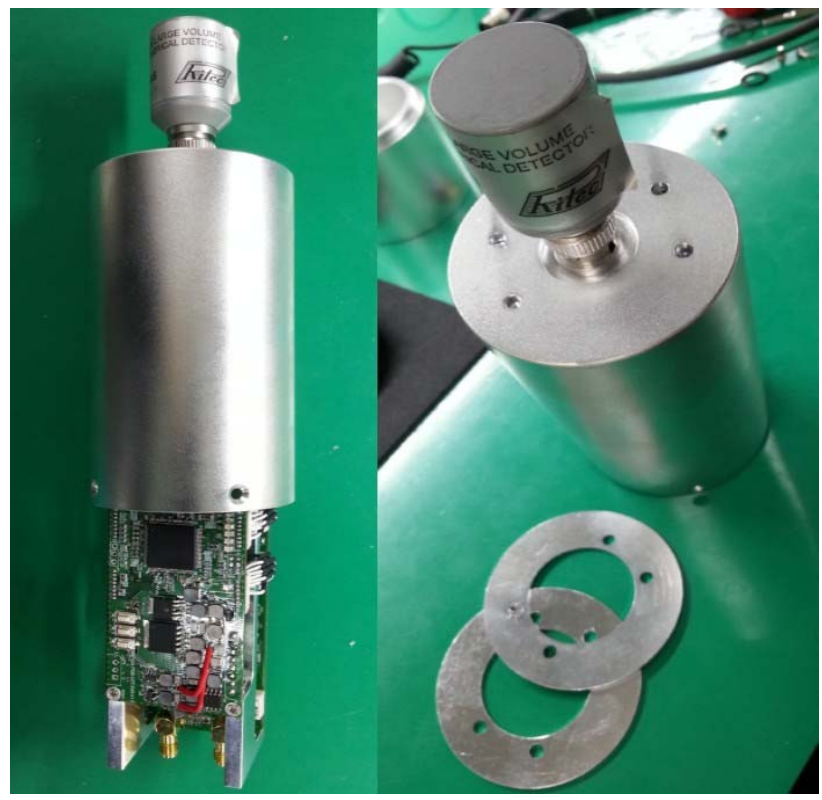

Fig. 5. A cylindrical body of a CZT detector.

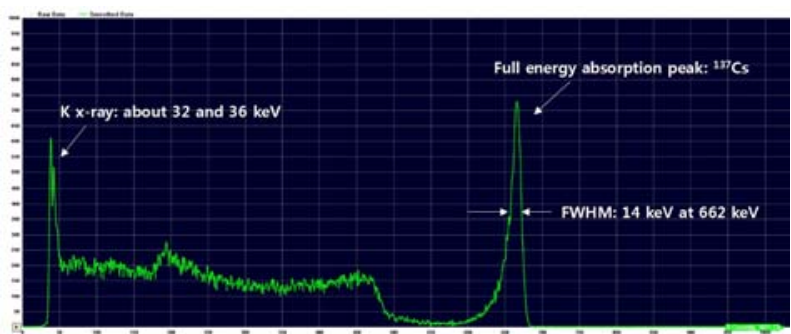

Fig. 6. Measured energy spectrum for a point source of ${ }^{137} \mathrm{Cs}$.

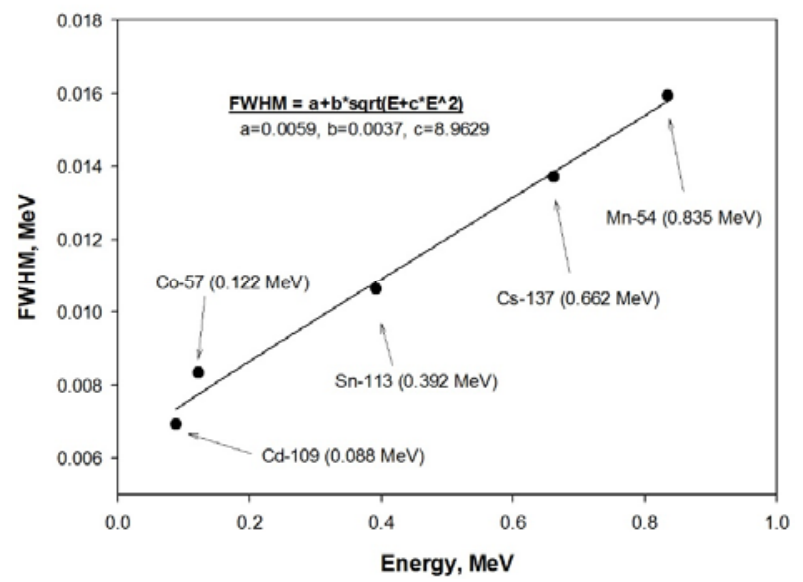

Fig. 7. Energy resolution for the developed CZT detector.

apply to the in-situ measurement of ${ }^{131} \mathrm{I},{ }^{134} \mathrm{Cs}$, and ${ }^{137} \mathrm{Cs}$ in the nuclear accident response.

\section{SYSTEM CONTROL UNIT}

The system control unit was designed to operate the CZT detector, monitor the detector, battery, and interface status, and check the measured results of the dose rate and radioactivity. In the system control part, as shown in Fig. 8, the GPS, internal battery and DC input, LCD, control buttons, and several interfaces, such as Bluetooth, USB (universal serial bus), and RS232 (recommended standard-232), were arranged with the on-board type. In the interface, Bluetooth and RS232 are dedicated to identify and remotely control the measured energy spectrum and the dose rate, and the USB is used to upgrade the system and analysis algorithm in the CZT detector. Several buttons and LED (light emitting diode) lamps were mounted to monitor the status of the battery, the detector operation, and the Bluetooth interface. In addition, by mounting a tripod to the detector body, the remote control for all processes was achieved using the Bluetooth interface

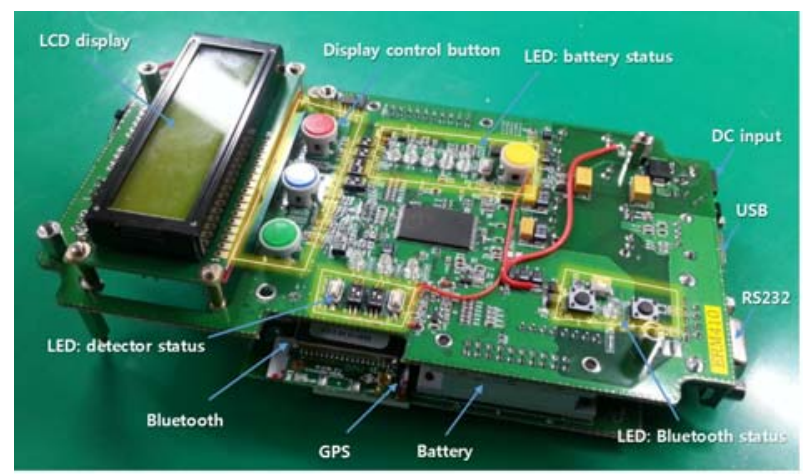

Fig. 8. Developed system control unit with an on-board type.

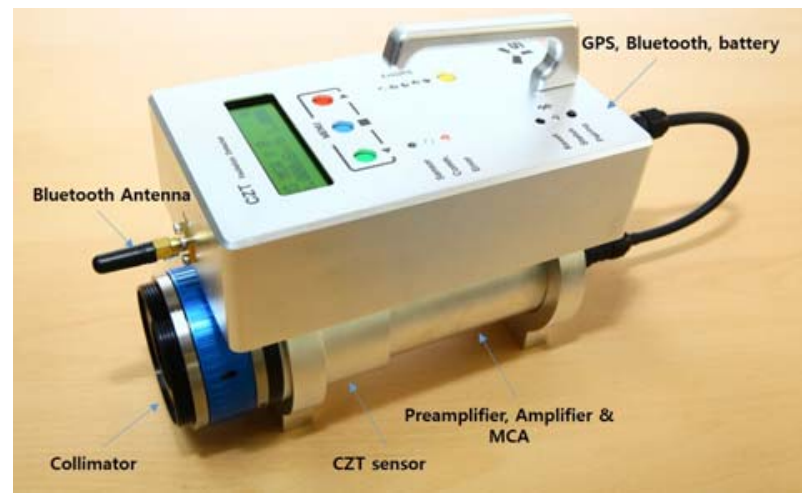

Fig. 9. Developed in-situ gamma-ray spectrometer based on the CZT detector.

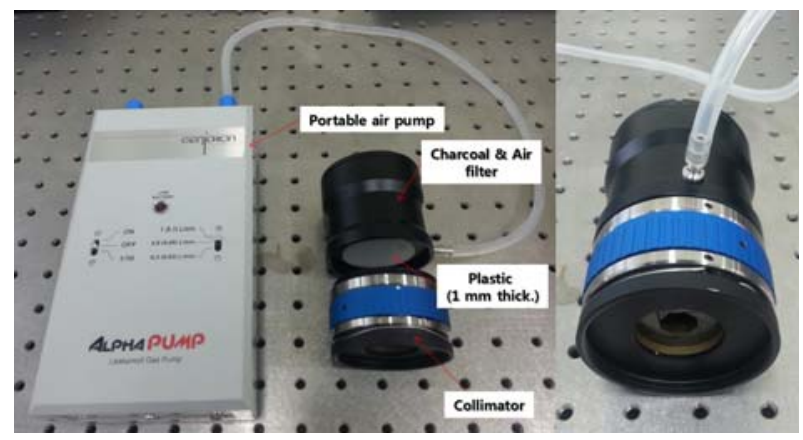

Fig. 10. The airborne dust sampler and collimator. 
between the detector and a laptop. Fig. 9 shows the final version of the developed in-situ gamma-ray spectrometer based on the CZT detector.

\section{COLLIMATOR AND AIRBORNE DUST SAMPLER}

For a rapid response to a nuclear accident through in-situ gamma-ray spectrometry, several collimators with different inner radii should be prepared according to the level of the ambient dose rate. This makes a quick response impractical and thus a collimator was then developed to have a function of controlling the inner radius with the same method used in a commercial camera lens. The attenuation ratio according to the inner radius, which was calculated from the Monte Carlo simulation, was marked on the outer scale ring of the collimator. An airborne dust sampler, which consists of an air filter and charcoal filter, was modified to attach to the front of a collimator, as shown in Fig. 10. This sampler is for the sampling airborne dust in the field survey for the emergency response with it mounted on the CZT detector. Therefore, the continuous analysis of the airborne dust will be conducted through the in-situ gamma-ray spectrometry using the CZT detector.

\section{CONCLUSION}

To determine the source and its amount of contaminants and divide the contaminated areas in the response of the nuclear accident, the information on the ambient dose rate in the scene of the accident and the radioactivity of the contaminants should be quickly, efficiently, and accurately corrected by mobilizing possi- ble methods. In this study, for the above purpose, the hand-held CZT detector was developed to make the in-situ gamma-ray spectrometry and its performance was checked to have a good energy resolution. In addition, the collimator and the airborne dust sampler were developed and mounted to the developed CZT detector for a quick and efficient response to a nuclear accident.

\section{ACKNOWLEDGEMENT}

This work was performed under the auspices of the Ministry of Science, ICT and Future Planning (MSIP) of Korea, NRF contract No. 2012M2A8A4025915.

\section{REFERENCES}

1. Cho G. Gamma-ray detectors for nuclear medical imaging instruments. Nucl. Med. Mol. Imaging. 2008;42(2):88-97.

2. Park SH, Lee NH, Lee SM, Ha JH, Lee JH, Kim HS, Kim YK. New concept of CZT-based hand-held radioisotope identifier. Appl. Radiat. Isot. 2009;67:1471-1475.

3. Gonzalez R, Perez JM, Vela O, Burgos E. Performance comparison of a large volume CZT semiconductor detector and a $\mathrm{LaBr} 3(\mathrm{Ce})$ scintillator detector. IEEE Trans. Nucl. Sci. 2006;53(4):24092415.

4. Ji YY, Chung KH, Lee W, Choi SD, Kim CJ, Kang MJ, Park ST. Development of the IRIS collimator for the portable radiation detector and its performance evaluation using the MCNP code. J. Nucl. Fuel. Cycle Waste Technol. 2015;13(1):417-423.

5. Gilmore G, Hemingway JD. Practical gamma-ray spectrometry. $2^{\text {nd }}$ ed. New York; John Wiley \& Sons, 1995:73-83. 
